\title{
PID Controller Design for Non-Minimum Phase Time-Delay Systems Using Optimization Methods
}

\author{
${ }^{1}$ B.Amarendra Reddy, ${ }^{2}$ M.Anil Kumar, ${ }^{3}$ K.Anusha, ${ }^{4}$ G.Ananda Rao \\ Department of Electrical Engineering, Andhra University (A) \\ Email: bamarendrareddy@yahoo.com,anilkumarmarada227@gmail.com, anushakanu95@gmail.com, \\ gopisettiananda@gmail.com
}

Received: 10 $^{\text {th }}$ December 2017, Accepted: 17th January 2018, Published: 28th February 2018

\begin{abstract}
Non-minimum phase systems are difficult to control with conventional methods because of their inherent characteristics of undershoot. The complexity in control of such systems increases when time delay is added. These systems will exhibit undershoot in time-response and oscillations in the high frequency region in magnitude frequency response. This paper focuses on design of PID controller for nonminimum phase time-delay systems under the disturbance and noise conditions. The PID controllers for these systems are designed by using Magnitude-Optimum Multiple Integration and numerical optimization methods. Mathematical analysis is developed using numerical optimization technique for non-minimum phase time delay systems. By using these methods the PID controllers are obtained mathematically. The effectiveness of PID controllers resulted using these methods compared in MATLAB environment by considering NMP time-delay systems.
\end{abstract}

Keywords: Magnitude optimum and multiple integration method, Numerical optimization approach, Non minimum phse systems, Controllr parameters.

\section{Introduction}

There have been great amount of research work on the tuning of PID controllers. In this paper, two approaches are given for the controller design of non-minimum phase systems. PID controllers have been used for a long time. Taylor developed the first PID controller [12]. But the problem is occurred how to tune a PID controller. At that time Ziegler and Nichols discover the famous Ziegler and Nichols tuning rules. These rules are still widely used. This is why so many different tuning rules have been developed which are based on the same tuning procedure. In this paper discussed about the controller design of the non-minimum phase systems by using magnitude optimum and multiple integration and numerical optimization approach.

In this paper the procedural steps for design of a controller for Non-minimum phase time-delay systems using MOMI are discussed in section II. Design of controllers using Numerical optimization is discussed in section III. To validate these concepts simulation results are presented in section VI.

\section{MAGNITUDE OPTIMUM AND MULTIPLE INTEGRATION METHOD.}

Consider a stable process control system described using equation (1)

$G_{P}(s)=K_{P R} \frac{1+b_{1} s+b_{2} s^{2}+\ldots .+b_{m} s^{m}}{1+a_{1} s+a_{2} s^{2}+\ldots .+a_{n} s^{n}} e^{-s T_{d e l}}$

Using the taylor series expansion the time delay can be defined using (2).

$\mathrm{e}^{-s T_{d e l}}=1-s T_{d e l}+\frac{\left(s T_{d e l}\right)^{2}}{2 !}-\frac{\left(\mathrm{s} T_{d e l}\right)^{3}}{3 !}+\ldots$

The PID controller ( $G_{C}=$ feedback controller) is defined using equation (3)

$G_{C}(s)=\left[\frac{K_{p} s+K_{i}+K_{d} s^{2}}{s\left(s+T_{f}\right)}\right]$

The closed-loop transfer function ' $G_{C L}$ ' of an unity feedback control system shown in Fig.(1). is defined using (4).

$G_{C L}=\frac{Y(s)}{R(s)}=\frac{G_{c}(s) G_{p}(s)}{1+G_{c}(s) G_{p}(s)}$

(4)

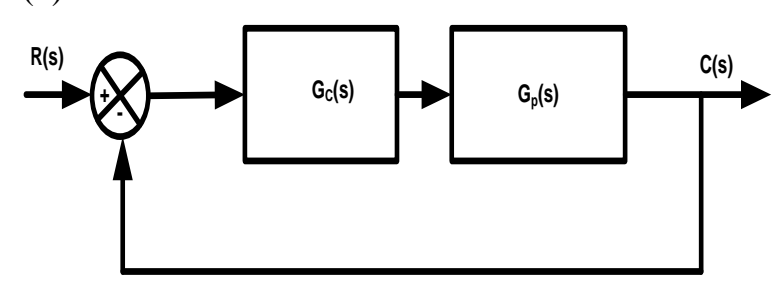

Fig.(1). Unity feedback control system

(i) Magnitude Optimum (Mo) Criteria:

The objectives of a controller design for any system is to ensure the system output to follow the reference signal. The main idea behind this magnitude-optimum method is to maintain the closed-loop magnitude frequency response $G_{C L}$ from reference to process output as flat, and as close to unity as possible over a large band width [1]. Here, the objective is to find a PID controller that makes the frequency response of the closed-loop amplitude as possible to unity over wide range of frequencies in the low frequency region. These requirements can be expressed mathematically as given in equations (5) and (6). 


$$
G_{C L}(0)=1 \quad(5) \quad \frac{d^{2 k}\left|G_{C L}(j \omega)\right|^{2}}{d \omega^{2 k}}=0 \text { towards }
$$

$\omega=0 ; \mathrm{k}=1,2, \ldots \ldots \ldots k_{\text {max }}$

This technique is called "Magnintude Optimum", "Modulus Optimum" ,"Betrags Optimum". The advantage of this method is to make closed-loop step response non oscillatory and response time is very fast [11]-[13]. Let the closed-loop transfer function is described using (7).

$G_{C L}=\frac{f_{0}+f_{1} s+f_{2} s^{2}+f_{3} s^{3}+\ldots}{e_{0}+e_{1} s+e_{2} s^{2}+e_{3} s^{3}+\ldots}$

To make the closed-loop frequency response magnitude unity over a wide frequency range and also to obey derivatives of closed-loop system $G_{C L}$ as defined in equation (6) results in a recursive equation (8)

$$
\sum_{i=0}^{2 n}(-1)^{i+n}\left(f_{i} f_{2 n-i} e_{0}^{2}-e_{i} e_{2 n-i} f_{0}^{2}\right)=0 ; \mathrm{n}=1,2,3 .
$$

The PID controller parameters $K_{p}, K_{i}, K_{d}$ are obtained by solving solving first three expressions resulted from equation(8) by substituting $n=1,2,3$. Solving the three equations, it is observe that $K_{p}, K_{i}, K_{d}$ are functions of $K_{p r}, a_{1}, a_{2}, a_{3}, a_{4}, a_{5}, b_{1}, \ldots \ldots . b_{5}, T_{\text {delay }}, T_{f}$.

Observations of Magnitude Optimum Method::

1.The PID controller parameter solution involves complicated expressions and they would cover several papers.

2.To calculate PID controller parameters only $a_{1}$ to $a_{5}, b_{1}$ to $b_{5} . T_{\text {delay }} K_{p r}$ needed even though process transfer function can be of higher order.

3.If process transfer function is not available estimating these parameters may be difficult.

4.Some times assumption of dynamic model in the required format for higher order system may lead to model uncertainty or modelling error. In such cases accurate model for a system may not possible.

(ii) Magnitude Optimum Multiple Integration Method ::

These difficulties in obtaining PID controller parameters can be avoided by using the concept of movements from identification theory using Magnitude optimum Multiple Integration method(MOMI).

According to MOMI tuning method the plant defined using equation (1) can be approximated using equation (9) Where $A_{i}(\mathrm{i}=0,1,2, \ldots .$.$) represents$ time-weighted integrals of the process impulse response. The coefficients $A_{i}$ 's can be obtained by measurement of areas which are calculated from process open-loop step response. These areas $A_{i}$ 's can be obtained by integrating the process input $\mathrm{U}(\mathrm{t})$ and process output $\mathrm{Y}(\mathrm{t})$ after applying during the change of process steady-state.
$G_{P}(s)=A_{0}-A_{1} s+A_{2} s^{2}-A_{3} s^{3}+\ldots \ldots .$,

(9)

where $A_{0}=K_{P R}$

$$
\begin{aligned}
& \mathrm{A}_{1}=\mathrm{y}_{1}(\infty)=\mathrm{K}_{\mathrm{PR}}\left(\mathrm{a}_{1}-\mathrm{b}_{1}+\mathrm{T}_{\text {del }}\right) \\
& A_{2}=y_{2}(\infty)=K_{P R}\left[b_{2}-a_{2}-T_{d e l} b_{1}+\frac{T_{d e l}^{2}}{2}\right]+A_{1} a_{1} \\
& A_{3}=y_{3}(\infty)=K_{P R}\left[a_{3}-b_{3}+T_{d e l} b_{2}+\frac{T_{d e l}^{2} b_{1}}{2}+\frac{T_{d e l}^{3}}{3 !}\right]+A_{2} a_{1}-A_{1} a_{2} \\
& A_{4}=y_{4}(\infty)=K_{P R}\left[\begin{array}{l}
b_{4}-a_{4}-T_{d e} b_{3}+\frac{T_{d e l}^{2} b_{2}}{2}- \\
\frac{T_{d e l}^{3} b_{1}}{3 !}+\frac{T_{d e l}^{4}}{4 !}
\end{array}\right]+A_{3} a_{1}-A_{2} a_{2}+A_{1} a_{3} \\
& A_{5}=y_{5}(\infty)=K_{P R}\left[\begin{array}{l}
a_{5}-b_{5}+T_{d e l} b_{4}-\frac{T_{d e l}^{2} b_{3}}{2}+ \\
\left.\frac{T_{d e l}^{3} b_{2}}{3 !}-\frac{T_{d e l}^{4} b_{1}}{4 !}+\frac{T_{d e l}^{5}}{5 !}\right]+A_{4} a_{1}-A_{3} a_{2}+A_{2} a_{3}-A_{1} a_{4}
\end{array}\right.
\end{aligned}
$$

To simplify the computation of PID controller parameters the derivative filter coefficient is considered be a part of process and considered as $G_{p}{ }^{*}(s)$.

$G_{p}^{*}(s)=\frac{G_{p}(s)}{1+T_{f}}$

To include the derivative filter coefficients to be a part of $G_{P}$ recalculate $A_{0}^{*}, A_{1}^{*}, A_{2}^{*} \ldots \ldots \ldots$

$$
\begin{aligned}
& A_{0}^{*}=A_{0} \\
& A_{1}^{*}=A_{1}+A_{0} T_{f} \\
& A_{2}{ }^{*}=A_{2}+A_{1} T_{f}+A_{0} T_{f}{ }^{2} \\
& A_{3}^{*}=A_{3}+A_{2} T_{f}+A_{1} T_{f}{ }^{2}+A_{0} T_{f}{ }^{3} \\
& A_{4}^{*}=A_{4}+A_{3} T_{f}+A_{2} T_{f}{ }^{2}+A_{1} T_{f}{ }^{3}+A_{0} T_{f}{ }^{4} \\
& A_{5}^{*}=A_{5}+A_{4} T_{f}+A_{3} T_{f}{ }^{2}+A_{2} T_{f}{ }^{3}+A_{1} T_{f}{ }^{4}+A_{0} T_{f}{ }^{5} \\
& G_{C}{ }^{*}=\left(\frac{K_{I}+K_{p} s+K_{d} s^{2}}{s}\right)
\end{aligned}
$$

Obtain the coefficients $e_{0}, e_{1}, e_{2} \ldots \ldots f_{0}, f_{1} \ldots \ldots$ by substituting $G_{C}{ }^{*}$ as given in equation (11) and $G_{P}(s)$ as given in equation (9). Solve the first three equations of equation (8) and the PID controller parameters $K_{p}, K_{i}, K_{d}$ can be obtained using (12).

$$
\left[\begin{array}{l}
K_{i} \\
K \\
K_{d}
\end{array}\right]=\left[\left(\begin{array}{ccc}
-A_{1} & A_{0} & 0 \\
-A_{3} & A_{2} & -A_{1}-T_{f} A_{0} \\
-A_{5} & A_{4} & -A_{3}-T_{f}^{2} A_{1}-T_{f}^{3} A_{0}
\end{array}\right]\right]^{-1} \times\left[\begin{array}{c}
-0.5 \\
0 \\
0
\end{array}\right]
$$

Numerical Optimization Method

Numerical optimization presents a comprehensive and up to date description of the most effective methods in optimization of continuous-time systems. Design the PID controller for a nonminimum phase time-delay system using numerical optimization method. Here, the optimization problem is formulated based on the maximization of the shortest distance between the critical point to Nyquist curve of open-loop transfer function[14]. 
The distance between critical point to the Nyquist curve is mathematically defined in equation (13). The controller parameters $\mathrm{Kp}, \mathrm{Ki}, \mathrm{Kd}$ are identified such that the distance from the critical point to the Nyquist curve is maximized. This maximization helpful to achieve the good stability margins , robustness to model uncertainties and also help to reject the disturbances i.e., this maximization minimizes the sensitivity.

$$
1+L(s)
$$

The procedural steps for computation of PID controller parameters using this numerical optimization is enumerated as follows.

(i) The open loop transfer function of nonminimum phase time-delay system with standard PID controller is

$L(s)=\frac{k\left(1+k_{p} T_{i} s+k_{d} T_{i} s^{2}\right)\left(1-P_{0} s\right) e^{-t_{0} s}}{T_{i} s\left(s^{2}+a_{1} s+a_{0}\right)}$, with $T_{i}=1 / k_{i}$.

(ii) Formulate the characteristic equation of nonminimum phase time-delay system by assuming the approximation to the time delay as

$$
\begin{aligned}
& e^{-t_{0} s}=\frac{1}{\left(1+t_{0} s\right)} . \\
& s^{4}+s^{3}\left(\frac{1+a_{1} t_{0}-k k_{d} P_{0}}{t_{0}}\right)+s^{2}\left(\frac{a_{1}+a_{0} t_{0}+k k_{d}-k k_{p} P_{0}}{t_{0}}\right)+ \\
& s\left(\frac{k k_{p} T_{i}-k P_{0}+a_{0}}{T_{i} t_{0}}\right)+\frac{k}{T_{i} t_{0}}=\rho(s)
\end{aligned}
$$

(iii) The characteristic equation is of the form

$$
\begin{aligned}
& \rho(s)=(s+a)^{2}\left(s^{2}+2 \xi \omega_{0} s+\omega_{0}^{2}\right) \text { i.e. } \\
& \rho(s)=s^{4}+s^{3}\left(2 \xi \omega_{0}+2 a\right)+s^{2}\left(\omega_{0}^{2}+4 a \xi \omega_{0}+a^{2}\right) \\
& +s\left(2 \omega_{0}^{2} a+2 a^{2} \xi \omega_{0}\right)+a^{2} \omega_{0}^{2}
\end{aligned}
$$

(iv) Comparing the equations (14) and (15)

$a=\frac{1}{2} a_{1}+\frac{1}{2 t_{0}}-\frac{k k_{d} P_{0}}{2 t_{0}}-\xi \omega_{0}$

$k_{p}=\frac{\left(2\left(a \xi+\omega_{0}\right) a \omega_{0} t_{0}\right)+a^{2} \omega_{0}^{2} P_{0} t_{0}-a_{0}}{k}$

$k_{i}=\frac{a^{2} \omega_{0}^{2} t_{0}}{k}$

$k_{d}=\frac{1+a_{1} t_{0}-2 \xi \omega_{0} t_{0}-2 a t_{0}}{k P_{0}}$

(v) The closed-loop stability impose constraint on 'a' i.e., $a>0$

To obey this condition $\frac{1}{\xi \omega_{0}}\left(\frac{1}{2} a_{1}+\frac{1}{2 t_{0}}-\frac{k k_{d} P_{0}}{2 t_{0}}\right)>1$

The above inequality is satisfied for

$\frac{1}{\xi \omega_{0}}\left(\frac{1}{2} a_{1}+\frac{1}{2 t_{0}}-\frac{k k_{d} P_{0}}{2 t_{0}}\right)=b$

(vi) With $\mathrm{b}>1$. One can choose the settling time with desired damping $\xi=\xi_{m}$ for a specific nonminimum phase time delay system.

$$
\begin{aligned}
& \omega_{0}=\frac{1}{\xi_{m} b}\left(\frac{1}{2} a_{1}+\frac{1}{2 t_{0}}-\frac{k k_{d} P_{0}}{2 t_{0}}\right) \\
& a=\frac{1}{2} a_{1}+\frac{1}{2 t_{0}}-\frac{k k_{d} P_{0}}{2 t_{0}}-\xi_{m} \omega_{0}
\end{aligned}
$$

(vii) Obtain the PID controller parameters using the following formulae.

$$
\begin{aligned}
& k_{p}=\frac{\left(2\left(a \xi_{m}+\omega_{0}\right) a \omega_{0} t_{0}\right)+a^{2} \omega_{0}^{2} P_{0} t_{0}-a_{0}}{k} \\
& k_{i}=\frac{a^{2} \omega_{0}^{2} t_{0}}{k} \\
& k_{d}=\frac{1+a_{1} t_{0}-2 \xi_{m} \omega_{0} t_{0}-2 a t_{0}}{k P_{0}}
\end{aligned}
$$

Therefore, the optimization problem can be defined as

Determine $\mathrm{Kp}, \mathrm{Ki}, \mathrm{Kd}$ such that $\max _{b>1}\left\{\begin{array}{l}\text { minimize the distance between the Nyquist curve } \\ \text { of openloop transfer unction from the critical point }\end{array}\right\}$

Where $L(s)=\frac{k\left(1+k_{p} T_{i} s+k_{d} T_{i} s^{2}\right)\left(1-P_{0} s\right) e^{-t_{0} s}}{T_{i} s\left(s^{2}+a_{1} s+a_{0}\right)}$

Subjected to equality constrains

$$
\begin{aligned}
& \omega_{0}=\frac{1}{\xi_{m} b}\left(\frac{1}{2} a_{1}+\frac{1}{2 t_{0}}-\frac{k k_{d} P_{0}}{2 t_{0}}\right) \\
& a=\frac{1}{2} a_{1}+\frac{1}{2 t_{0}}-\frac{k k_{d} P_{0}}{2 t_{0}}-\xi_{m} \omega_{0} \\
& k_{p}=\frac{\left(2\left(a \xi_{m}+\omega_{0}\right) a \omega_{0} t_{0}\right)+a^{2} \omega_{0}^{2} P_{0} t_{0}-a_{0}}{k} \\
& k_{i}=\frac{a^{2} \omega_{0}^{2} t_{0}}{k} \\
& k_{d}=\frac{1+a_{1} t_{0}-2 \xi_{m} \omega_{0} t_{0}-2 a t_{0}}{k P_{0}}
\end{aligned}
$$

\section{EXAMPLES.}

To show the effectiveness of these optimum based PID controller design methods, non-minimum phase time-delay systems are considered for simulation in MATLAB environment.

Example 1:

Consider the second-order non-minimum phase time delay system described using a transfer function $G_{1}(s)$.

$G_{1}(s)=\frac{(1-S) e^{-s}}{s^{2}+2 s+1}$

Case (a) : magnitude optimum and multiple integration method:

The detailed step-by step computation procedure to obtain PID controller parameters using magnitude optimum multiple integration method is given as below. From the given problem, A0 to A5 coefficients are obtained and the derivative filter coefficient is chosen as 0.01 . 
$K_{p r}=1, T_{d e l}=1$,

$A_{0}=1, A_{1}=4, A_{2}=18.5000, A_{3}=64.3333$,

$A_{4}=115.3750, A_{5}=166.4167$.

$T_{f}=0.01$

Using the equation (12) the PID controller parameters values are obtained as $K_{i}=0.2363, K_{p}=0.4454, K_{d}=0.1975$

Case(b): Numerical optimization method:

Using the expressions derived using numerical optimization technique for this non-minimum phase time delay system, the PID controller parameters are:

$a_{0}=1 ; a_{1}=2 ; p_{0}=1 ; t_{0}=1 ; k=1 ; \omega_{0}=1.3 ; b=1.4 ; \xi=0.75 ;$

$a=0.3900 ; K_{p}=0.4454 ; K_{i}=0.2363 ; K_{d}=0.1975$

Using these methods MOMI and NA, the controllers are designed. To show the effectiveness of these controllers simulations are performed in the MATLAB environment. It is observed from this simulation that these PID controllers are effectively rejecting the disturbances and noise entering into the system. From the magnitude sensitivity frequency response shown in Fig.(4)., it is observed that both controllers are effectively rejecting the disturbance. From the time-domain simulation shown in Fig.(2)., it is observed that when a step disturbance signal of magnitude 1 applied at 60 seconds at the process input these controllers are effectively regulating the system. Fig.(3). Shows the time response of $G_{1}(s)$ under noise conditions. From the magnitude complementary sensitivities graphs, it is observed, these PID controllers can effectively attenuate noise entering into the system. It shown effectively using the time domain simulation by injecting a high frequency noise signal at the output sensor. The Performance requirement on Complimentary Sensitivity function for $G_{1}(s)$ ishown in Fig.(5).

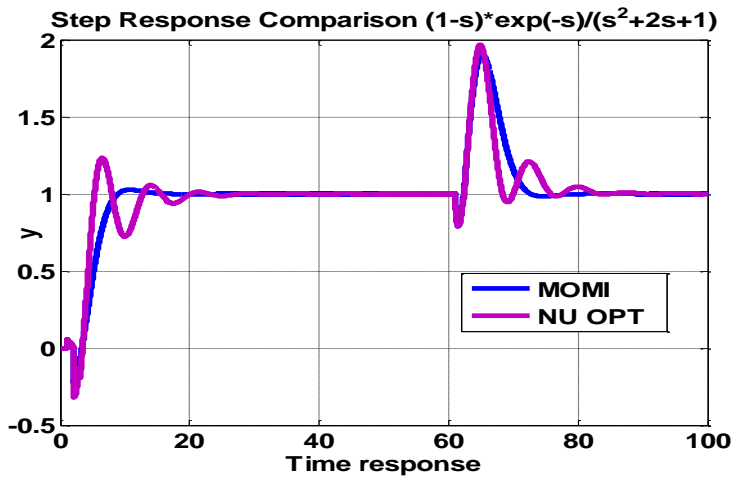

Fig.(2). Time response of $G_{1}(s)$ with MOMI and NA Controllers

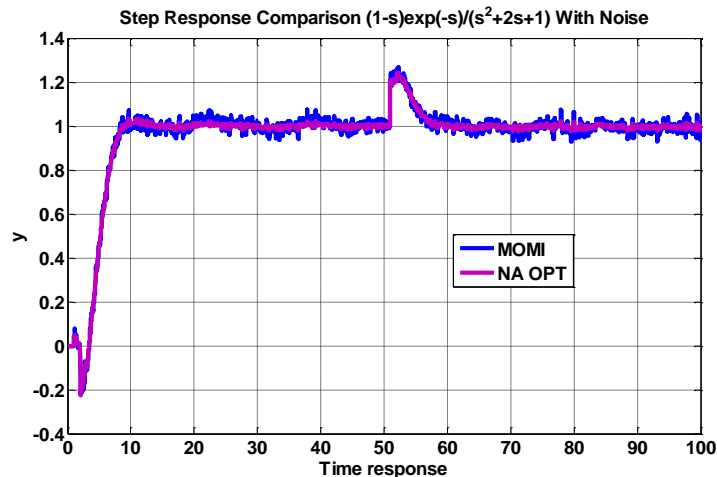

Fig.(3). Time response of $G_{1}(s)$ under noise conditions

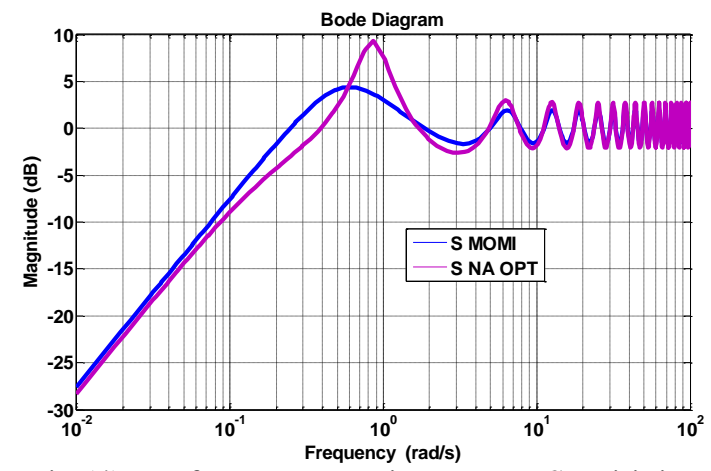

Fig.(4). Performance requirement on Sensitivity function for $\mathrm{G}_{1}(\mathrm{~s})$

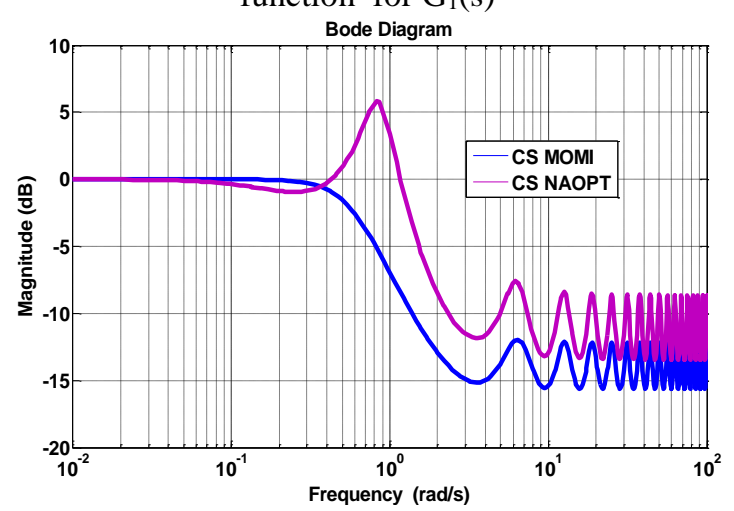

Fig.(5). Performance requirement on

Complimentary Sensitivity function for $\mathrm{G}_{1}(\mathrm{~s})$

Example 2:

Consider the second-order non-minimum phase time delay system described using a transfer function $G_{2}(s)$.

$G_{2}(s)=\frac{1.5(-0.2 s+1) e^{-0.2 s}}{s^{2}+2 s+1}$

Case (a) : magnitude optimum and multiple integration method:

The detailed step-by step computation procedure to obtain PID controller parameters using magnitude optimum multiple integration method is given as below. From the given problem, A0 to A5 coefficients are obtained and the derivative filter coefficient is chosen as 0.01 . 
$K_{p r}=1.5, T_{d e l}=0.2$,

$A_{0}=1.5, A_{1}=3.6, A_{2}=5.75, A_{3}=8.0$

$A_{4}=10.19, A_{5}=12.4$

$T_{f}=0.01$

Using the equation (12) the PID controller parameters values are obtained as $K_{i}=0.8081, K_{p}=1.6060, K_{d}=0.7894$

Case(b): Numerical optimization method:

Using the expressions derived using numerical optimization technique for this non-minimum phase time delay system the PID controller parameters are: $a_{0}=1 ; a_{1}=2 ; p_{0}=0.2 ; t_{0}=0.2 ; k=1.5$;

$\omega_{0}=0.9 ; b=4.6 ; \xi=0.75 ; a=2.2325$

$K_{p}=0.8203 ; K_{i}=0.5383 ; K_{d}=0.7900$

Using these methods MOMI and NA, the controllers are designed. To show the effectiveness of these controllers simulations are performed in the MATLAB environment. It is observed from this simulation that these PID controllers are effectively rejecting the disturbances and noise entering into the system. From the magnitude sensitivity frequency response shown in Fig.(8)., it is observed that both controllers are effectively rejecting the disturbance. From the time-domain simulation shown in Fig.(6)., it is observed that when a step disturbance signal of magnitude 1 applied at 60 seconds at the process input these controllers are effectively regulating the system. Fig.(7). Shows the time response of $G_{2}(s)$ under noise conditions.From the magnitude complementary sensitivities graphs, it is observed, these PID controllers can effectively attenuate noise entering into the system. It shown effectively using the time domain simulation by injecting a high frequency noise signal at the output sensor. The Performance requirement on Complimentary Sensitivity function for $\mathrm{G}_{2}$ (s) ishown in Fig.(9).

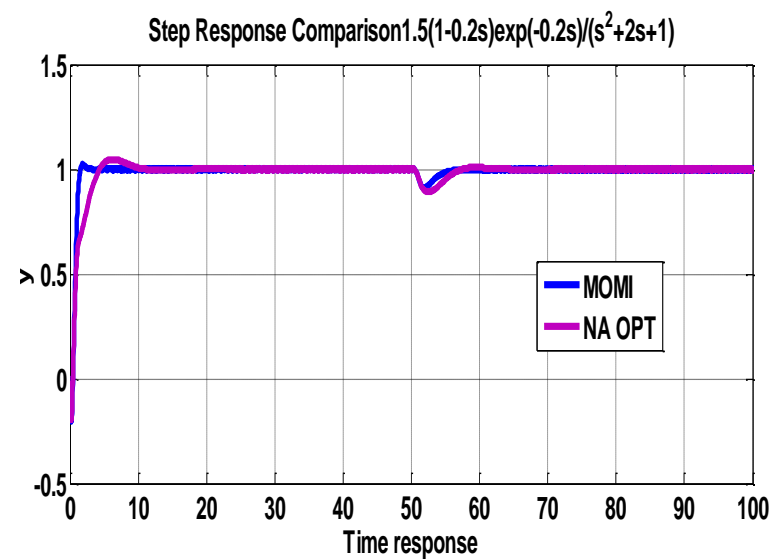

Fig.(6). Time response of $G_{2}(s)$ with MOMI and NA Controllers

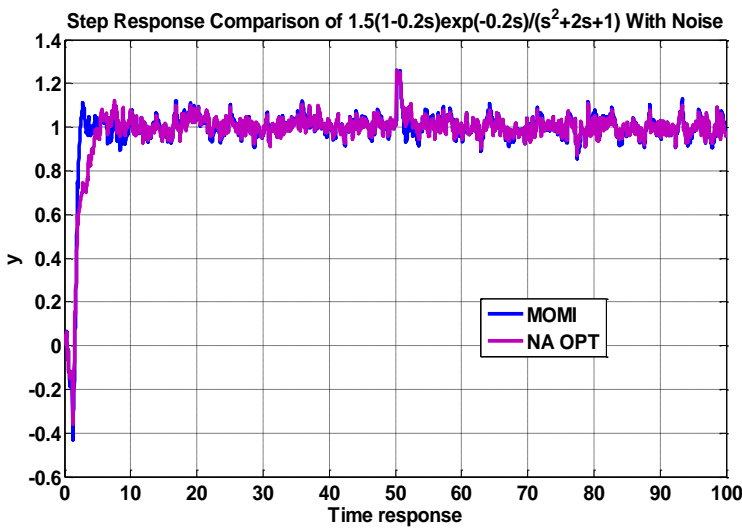

Fig.(7). Time response of $G_{2}(s)$ under noise conditions

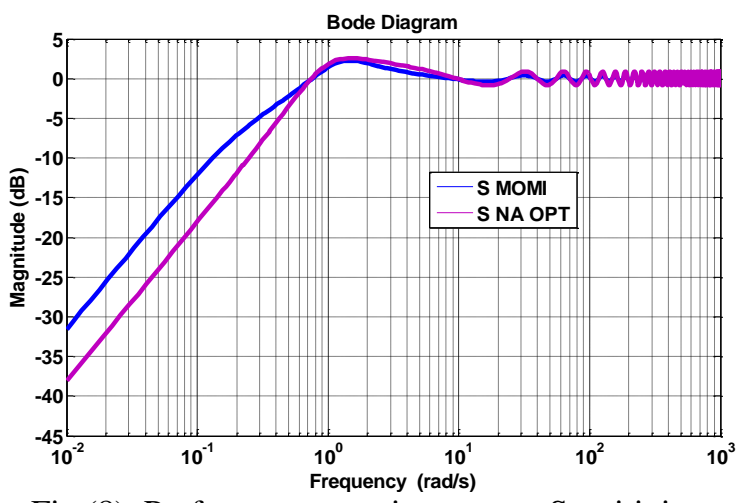

Fig.(8). Performance requirement on Sensitivity function for $\mathrm{G}_{2}(\mathrm{~s})$

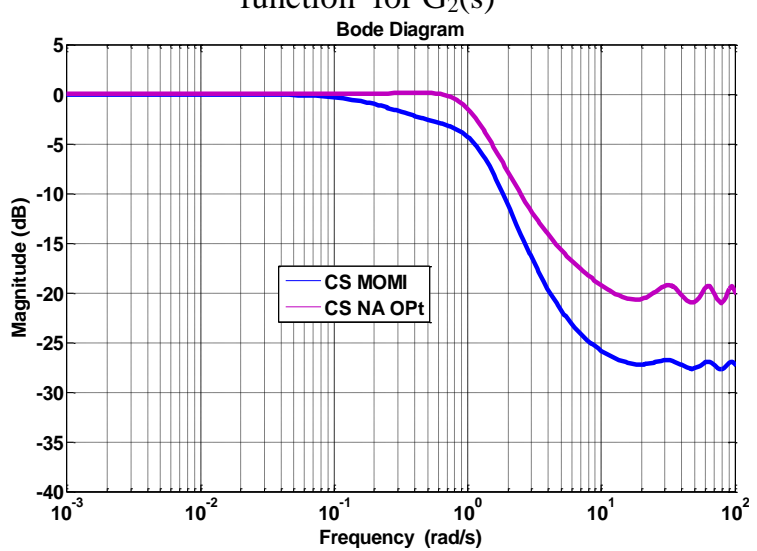

Fig.(9). Performance requirement on

Complimentary Sensitivity function for $\mathrm{G}_{2}(\mathrm{~s})$

Example 3:

Consider the second-order non-minimum phase time delay system described using a transfer function $G_{3}(s)$.

$G_{3}(S)=\frac{(1-s) e^{-s}}{s^{2}+3 s+2}$

Case (a): magnitude optimum and multiple integration method:

The detailed step-by step computation procedure to obtain PID controller parameters using MOMI method is given as below for this system. From the given problem, A0 to A5 coefficients are obtained and the derivative filter coefficient is chosen as 0.01 . 
$K_{p r}=1, T_{d e l}=1$,

$A_{0}=1, A_{1}=5, A_{2}=15.5000, A_{3}=41.1667$

$A_{4}=108.1250, A_{5}=283.2583$

$T_{f}=0.01$

Using the equation (12) the PID controller parameters values are obtained as

$K_{i}=0.2530, K_{p}=0.6651, K_{d}=0.1430$

Case(b): Numerical optimization method:

Using the expressions derived using numerical optimization technique for this non-minimum phase time delay system the PID controller parameters are:

$a_{0}=2 ; a_{1}=3 ; p_{0}=1 ; t_{0}=1 ; k=1$;

$\omega_{0}=0.5 ; b=4.5 ; \xi=0.75 ; a=1.3125$

$K_{p}=0.8702 ; K_{i}=0.5347 ; K_{d}=0.3250$

Using these methods MOMI and NA, the controllers are designed. To show the effectiveness of these controllers simulations are performed in the MATLAB environment. It is observed from this simulation that these PID controllers are effectively rejecting the disturbances and noise entering into the system. From the magnitude sensitivity frequency response shown in Fig.(12)., it is observed that both controllers are effectively rejecting the disturbance. From the time-domain simulation shown in Fig.(10)., it is observed that when a step disturbance signal of magnitude 0.5 applied at 60 seconds at the process input these controllers are effectively regulating the system. Fig.(11). Shows the time response of $G_{3}(s)$ under noise conditions. From the magnitude complementary sensitivities graphs, it is observed, these PID controllers can effectively attenuate noise entering into the system. It shown effectively using the time domain simulation by injecting a high frequency noise signal at the output sensor. The Performance requirement on Complimentary Sensitivity function for $G_{3}(s)$ ishown in Fig.(13).

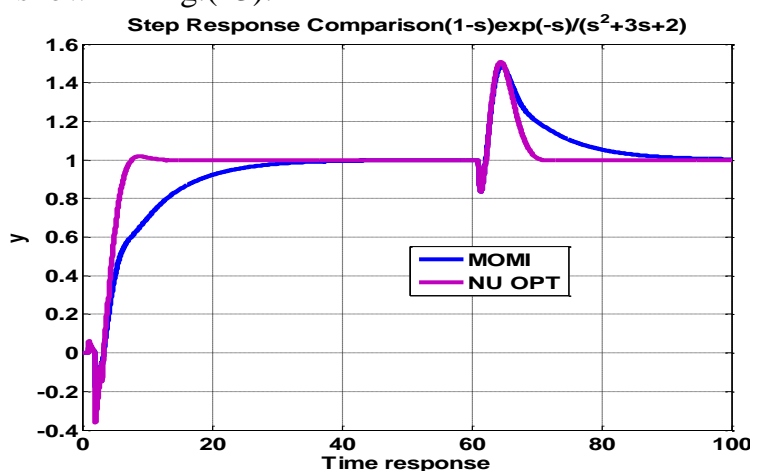

Fig.(10). Time response of $G_{3}(s)$ with MOMI and NA Controllers

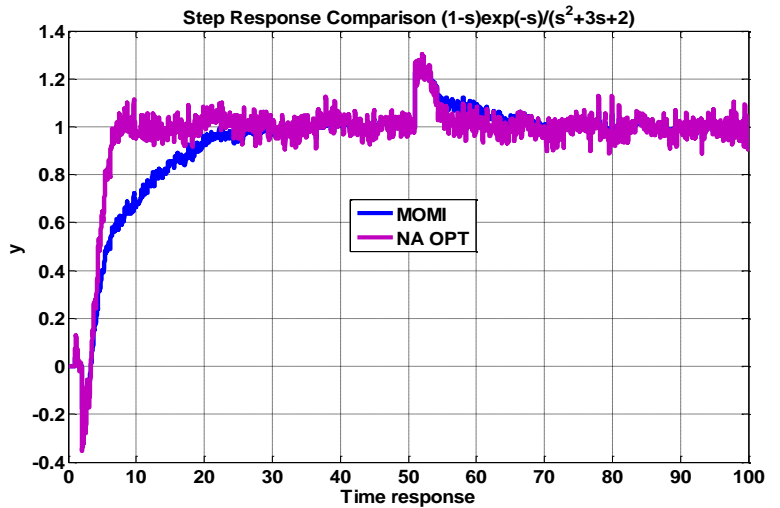

Fig.(11). Time response of $G_{3}(s)$ under noise

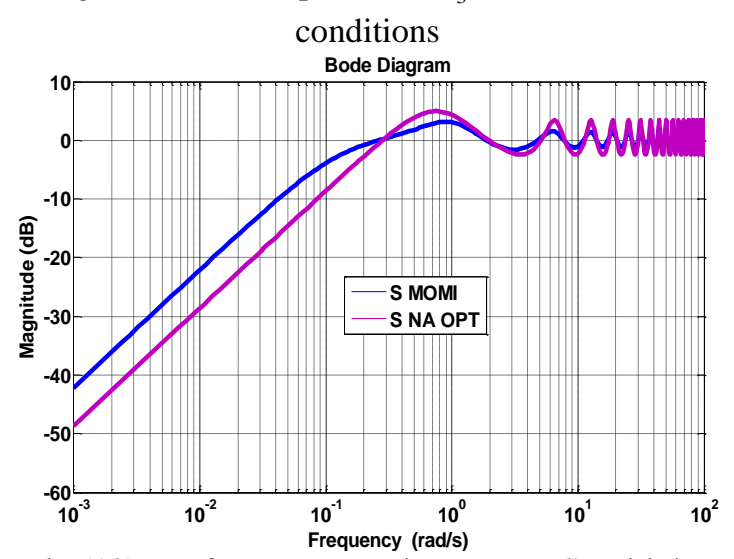

Fig.(12). Performance requirement on Sensitivity function for $\mathrm{G}_{3}(\mathrm{~s})$

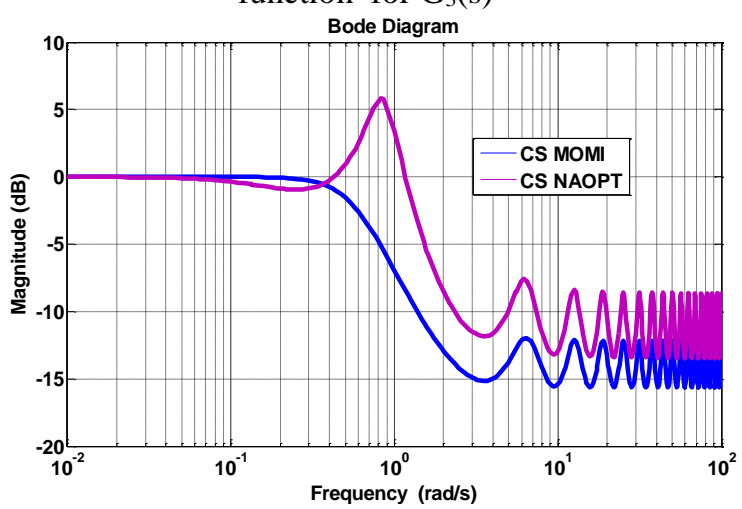

Fig.(13). Performance requirement on

Complimentary Sensitivity function for $\mathrm{G}_{3}(\mathrm{~s})$

\section{Conclusion}

In this paper mathematical analysis is developed using numerical optimization technique for nonminimum phase time delay systems. The PID controllers are designed for Non-minimum phase time-delay systems using the magnitude optimum multiple integration. These both methods are effectively rejecting disturbances. The effectiveness of these controllers in rejecting disturbances are shown in time-domain and also in frequency domain in the MATLAB environment. It is observed from these simulation results that MOMI method is more accurate compared to Numerical optimization. Magnitude sensitivity frequency responses using these controllers are shown for these systems. It is 
observed that these controllers are capable to reject disturbances and also attenuate the noice effectively.

\section{References}

1. Vrančić, D. (2008). MOMI Tuning Method for Integral Processes. Proceedings of the $8^{\text {Th }}$ Portuguese Conference on Automatic Control, Vila Real,

2. Astrom, K. J., Panagopoulos, H. \& Hagglund, T. (1998). Design of PI Controllers based on Non-Convex Optimization. Automatica, 34 (5), pp. 585601.

3. Ba Hli, F. (1954). A General Method for Time Domain Network Synthesis. IRE Transactions-Circuit Theory, 1 (3), pp. 2128.

4. Gorez, R. (1997). A survey of PID autotuning methods. Journal A. Vol. 38, No. 1, pp. 3-10.

5. Hanus, R. (1975). Determination of controllers parameters in the frequency domain. Journal A, XVI (3).

6. Huba, M. (2006). Constrained pole assignment control. Current Trends in Nonlinear Systems and Control, L. Menini, L. Zaccarian, Ch. T. Abdallah, Edts., Boston: Birkhauser, pp. 163-183.

7. Kessler, C. (1955). Uber die Vorausberechnung optimal abgestimmter Regelkreise Teil III.Die optimale Einstellung des Reglers nachdemBetragsoptimum.Regelungstechni k, Jahrg. 3, pp. 40-49.

8. Preuss, H. P. (1991). Model-free PIDcontroller design by means of the method of gain optimum (in German). Automatisierungstechnik, Vol. 39, pp. 1522.

9. Rake, H. (1987). Identification: Transientand frequency-response methods. In M. G. Singh(Ed.), Systems \& control encyclopedia; Theory, technology, applications. Oxford:Pergamon Press.

10. Strejc, V. (1960). Auswertung der dynamischen Eigenschaften von Regelstrecken bei gemessenen Ein- und Ausgangssignalen allgemeiner Art. Z. Messen, Steuern, Regeln, 3(1), pp. 7-10

11. Umland, J. W. \& M. Safiuddin (1990). Magnitude and symmetric optimum criterion for the design of linear control systems: what is it and how does it compare with the others? IEEE Transactions on Industry Applications, 26 (3), pp. 489-497.

12. Ali Akbar Jalali, Shabnam Khosravi(may 2011) Tuning of FOPID Controller Using Taylor Series Expansion

13. (13)Astrom,K.J., andHagglund(1995).PID controllers:theory tuning.instrumnt society of amera. $2^{\text {nd }}$ edition.

14. R.Toscano(31, march, 2004) (A simple robust PI/PID ontroller design via numerical optimization approach). 